\title{
Estudio preliminar del perfil comparativo del control financiero en jóvenes universitarios
}

\section{Preliminary study of the comparative profile of financial control in university young people}

\author{
Jorge Palacios ${ }^{1}$ \\ Leonardo Soler ${ }^{2}$ \\ ${ }^{1}$ Facultad de Psicología, Universidad del Valle de México. \\ ${ }^{2}$ Facultad de Estudios Superiores Zaragoza, Universidad Nacional Autónoma de México
}

\begin{abstract}
Resumen: El objetivo de la presente investigación fue obtener evidencias de validez y confiabilidad de la escala de locus de control financiero, además de analizar diferencias en variables estructurales. Participaron en el estudio 300 universitarios, 110 hombres y 190 mujeres, entre 18 y 59 años de edad con una media de $21.74(D E=6.7)$ de la Zona Metropolitana de la Ciudad de México. El locus de control se evaluó con ítems que reflejan que obtener dinero es contingente con sus acciones. Los resultados muestran las propiedades psicométrica de un instrumento que incorpora tres factores referentes al locus de control: interno, afectivo y externo. Además los hallazgos sugieren que los universitarios tienen un alto locus de control financiero interno y afiliativo, diferenciado por sexo, tipo de carrera y nivel de eficiencia laboral. La discusión analiza la utilidad de los resultados dentro de la sociocultura mexicana, es decir, que lograr tener dinero es atribuido a causas afectivas, fuentes externas, así como por la decisión propia de tener dinero, lo que tiene implicaciones al momento de buscar nuevos ingresos y las contingencias para tenerlo.
\end{abstract}

Palabras clave: psicología económica, locus de control, finanzas personales, jóvenes, evaluación psicológica

\begin{abstract}
The objective of the present research was to obtain evidences of validity and reliability of the scale of locus of financial control, obtaining differences in structural variables. The study involved 300 university students, 110 men and 190 women, between 18 and 59 years old $(M=$ 21.74; $S D=6.7$ ) from the Metropolitan Zone of Mexico City. The locus of control was assessed with items that reflect that obtaining money is contingent on its actions. The results show the psychometric properties of an instrument that incorporates three factors related to the locus of control: internal, affective and external. Additionally, the findings suggest that university students have a higher level of internal and affiliative financial locus of control, differentiated by sex, type of career and level of work efficiency. The discussion analyzes the utility of the results within the Mexican socioculture, that having money is attributed to affective causes, external sources, as well as the decision to own money, which has implications when seeking new income and the contingencies to have it.
\end{abstract}

Key Words: economics psychology, locus of control, personal finance, youths, psychological assessment

Cómo citar este artículo:

Palacios. J. \& Soler, L. (2017). Estudio preliminar del perfil comparativo del control financiero en jóvenes universitarios. Ciencias Psicológicas, 11(2), 201-212. doi: https://doi.org/10.22235/cp.v11i2.1491

Correspondencia: Jorge Palacios, Universidad del Valle de México- Campus Querétaro. Blvd. Juriquilla no. 1000 A Del. Santa Rosa Jáuregui / CP 76230 / Querétaro, México, e-mail: drjpalacios81@gmail.com. Leonardo Soler, Universidad Nacional Autónoma de México,e-mail: lexhiveleonardo@gmail.com 


\section{Introducción}

Las personas cada día se esfuerzan en ejercer cierto grado de control sobre los hechos que consideran valiosos en su vida, consiguiendo así realizar acciones personales, familiares, sociales y financieras. Estas acciones recobran importancia dependiendo de las atribuciones de éxito o fracaso que le asignan los individuos en su vida cotidiana. Para entender estas atribuciones, la teoría del aprendizaje social de Rotter $(1966,1975$, 1990) describe que entre la situación y los reforzadores ambientales median factores cognitivos, se basa en el supuesto de que los factores cognoscitivos ayudan a determinar la manera en que las personas reaccionarán a los reforzadores de su ambiente y son las expectativas personales las que finalmente determinan el comportamiento.

El comportamiento de un individuo puede ser debido al entorno en el que vive o a la capacidad del individuo para modificar su situación, este rasgo de personalidad es nombrado locus de control, que describe dos formas de control del comportamiento a través del reforzamiento interno o externo y al grado en que un individuo considera que los reforzamientos son contingentes con su conducta (Díaz-Loving \& Andrade, 1984). Para Bandura (1994), el control, hace referencia a la posibilidad de dominar un acontecimiento según se localice el control dentro o fuera de uno mismo. De esta forma las personas han aprendido que la recompensa o el éxito dependen de sus acciones y del control que tienen sobre su vida, estas personas tiene un locus de control interno. Mientras que otras personas consideran que los eventos en su vida son determinados por factores fuera de su control como la suerte, el destino o las situaciones, estas personas se se dice que tienen un locus de control externo (Cázares \& Berridi, 2000; Perry \& Morris, 2005; Rotter, 1975, 1990; Rotter \& Mulry, 1965).

Diversos estudios (Diaz-Guerrero, 1994; García \& Reyes, 2000; Laborín, Vera, Durazo, \& Parra, 2008; Triandis \& Suh, 2002) han señalado que los países con culturas individualistas tienen un locus de control interno, mientras que en países con una cultura colectivistas el locus de control se externaliza. En primera instancia se consideró al locus de control como un constructo unidimensional, caracterizado por un continuo de dos polos, mientras más externa es una persona, menos interna será y a la inversa (Rotter,1975,
1990), sin embargo, una serie de estudios realizados en México (Correa, Bedolla, \& Reyes, 2006; Díaz-Loving et al., 1984; García et al., 2000; La Rosa, Díaz-Loving, \& Andrade, 1986; Vera \& Cervantes, 2000) han demostrado que el constructo de locus de control es multidimensional, encontrando entre 3 y 5 dimensiones (p.e. 1. locus de control interno, 2. locus de control externo/ aleatorio, 3. locus de control afectivo/ afiliativo, 4. locus de control social/ familiar y 5. locus de control personal). Al parecer el mexicano además de contar con los factores típicos de internalidad y externalidad incorpora una dimensión claramente afectiva en su control, ya que integra las cualidades de su cultura, al ser obediente afiliativo, afectuoso y cortés con los demás, adicionando un estilo de automodificación para adaptarse a su entorno (Díaz-Guerrero, 1994). Al considerar las características que tienen los mexicanos (Palacios \& Martínez, 2017) y su importancia para esta cultura, parece relevante que para la presente investigación se integré la dimensión afectiva / afiliativa (García et al., 2000; La Rosa et al.,1986 Vera \& Cervantes, 2000) dentro del locus de control financiero, como una forma de atribuirse a sí mismo las razones por las cuales se tienen buenas relaciones interpersonales y que facilita la obtención de metas (ingresos), gracias a las relaciones afectivas que establece la persona.

Por otra parte, el locus de control se ha aplicado a distintas situaciones como el escolar (Camacho, Moreno, \& Hernández, 1994; Gonzalez, Corral, \& Maytorena, 2002), el ambiental (Bustos, Flores, \& Andrade, 2004), el político (Bedolla \& Mena, 2004), las relaciones de pareja (Montero, Rivera, Reyes, \& Díaz-Loving, 2008), las conductas de riesgo (Riccio-Howe, 1991), el cuidado de la salud (Leong, Molassiotis \& Marsh, 2004; Stenström \& Adersson, 2000) y la económia (Plunkett \& Buehner, 2007), sin embargo, en México no se cuenta con evidencia que señale la aplicación del locus de control en las finanzas de las personas. Las finanzas personales son un tema relativamente reciente dentro de la psicología (Cruz, 2001; Quintanilla, 1998) y el presente estudio lo aborda desde la perspectiva de los jóvenes, considerando que las conductas individuales se basan en la historia de sus reforzamientos (Kimble, Hirt, Díaz-Loving, Hosh, Lucker, \& Zárate, 2002) y en las propias habilidades para controlar o modificar hechos importantes en la vida (Palacios, 2011). 
La investigación sobre la psicología económica se ha centrado en definir sus conceptos (Alejo, Rojas, \& Pérez-Acosta, 2008), entender la cognición social (Crusius Van Horen \& Mussweiler, 2012), el pensamiento económico (Amar, Abello, Denegri, \& Llanos, 2007), las actitudes hacia el endeudamiento (Boddinton \& Kemp, 1999; Denegri, Cabezas, Del Valle, González, \& Sepúlveda, 2012), la motivación financiera (Rowley, Lown, \& Piercy, 2012), el comportamiento del consumidor (Samuel-Lajeunesse \& Gil, 2014), las estrategias para gestionar dinero (Palacios, 2014), los rasgos de personalidad (Palacios, Bustos, \& Soler, 2015) y en algunos modelos (Rodríguez, 2006) se incluyen factores económicos, sociales y personales que inciden en el comportamiento de compra.

En cuanto a los factores personales, se destaca que los estudios basados en teorías del aprendizaje social han servido para explicar distintos comportamientos (Dijkstra \& De Vries, 2000; Palacios, 2010, 2015; Palacios \& Parrao, 2010; Wilson-Barlow, Hollins, \& Clopton, 2014). Desde esta perspectiva el comportamiento está determinado en gran medida por los eventos de la experiencia del individuo y de su consecuente interacción con su ambiente social (Ardila, 2011; Galindo \& Ardila, 2012; Lipina \& Colombo, 2009). Particularmente la investigación basada en estas teorías (Bandura, 2001; Rotter, 1966) consideran que el comportamiento de las personas se vincula con las expectativas de reforzamiento, la acción sobre el problema (autoeficacia) y el control sobre los refuerzos (locus de control interno - externo).

Dentro de los estudios sobre locus de control en la economía y las finanzas se encuentran aproximaciones en investigaciones como las de Furnham (1986) quien elabora una escala de locus de control de la conducta económica. Lindstrom \& Rosvall (2013) relacionaron un mayor estrés económico con la falta de locus de control interno. Por su parte Lachman y Weaver (1998), asocian el locus de control interno con una alta sensación de bienestar, además de señalar que el locus de control interno es benéfico para todos los niveles socioeconómicos. Los autores explican que a pesar de las adversidades propias de la situación de pobreza, hay personas que logran un alto nivel de motivación al logro, así como una sensación de control y esto redunda en su calidad de vida. Por otro lado, Haider y Naeem (2013), identificaron diferencias en el locus de control de estudiantes pakistaníes, quienes señalan que las mujeres mostraron un control externo, mientras que los hombres un control interno. En lo que respecta a hallazgos relacionados a universitarios, Baguma y Chireshe (2012) identificaron en estudiantes universitarios, que un locus de control económico interno es influido por la edad, mientras que el ser mujer y tener un locus de control económico interno predecía negativamente la creencia del poder de los otros para influenciar los propios resultados económicos.

Como se puede observar, las teorías del aprendizaje social han permitido entender distintos comportamientos a través de diversas investigaciones (Bandura, 2002; Gwaltney, Shiffman, Norman, Paty, Kassel, Gnys, Hickcox, \& Balanbis, 2001; Palacios, 2011; Palacios \& Bustos, 2012a, b; Palacios \& Bustos 2013; Palacios \& Ramírez, 2016; Stuart, Borland, \& McMurray, 1994). Estos estudios se han centrando en estudiar el efecto que tiene la autoeficacia sobre las acciones que realizan los individuos y en menor medida han estudiado el control que las personas poseen sobre las situaciones y las conductas necesarias para cambiar su situación de pobreza o bien lograr mantener mejores finanzas personales. Por otra parte, en los últimos años un sector de la población que se interesa por conocer sus prácticas financieras son los estudiantes universitarios, específicamente por el aumento creciente del endeudamiento considerando el riesgo que ello implica para su vida financiera y laboral (Denegri et al., 2012). Por tal motivo, los objetivos de la presente investigación fueron obtener evidencias de validez y confiabilidad de la escala de locus de control financiero, además de obtener diferencias en variables demográficas como, sexo, tipo de universidad, tipo de carrera y ocupación, así como en variables financieras (uso de tarjeta de crédito y nivel de eficiencia en el trabajo y/o académica), en una muestra de universitarios mexicanos.

\section{Materiales y Métodos}

\section{Participantes}

Se seleccionó una muestra no probabílistica de manera intencional de 300 universitarios, 110 hombres y 190 mujeres, con un rango de edad entre 18 y 59 años con una media de 21.74 
$(D E=6.7)$, de diferentes lugares de la Zona Metropolitana de la Ciudad de México. El 50.3\% eran de a una Universidad pública y $49.7 \%$ de una privada. El $54.7 \%$ pertenecían a las áreas biológicas, sociales y de la salud y el $45.3 \%$ restante eran de las áreas económicas y administrativas. Respecto a su ocupación se encontró que el $71 \%$ únicamente estudiaba, el $26.3 \%$ estudiaba y trabajaba, y sólo el $2.7 \%$ únicamente estaba trabajando al haber terminado sus estudios. Además se les preguntó sobre el uso de tarjetas de crédito, el $22.3 \%$ señaló que tenía tarjeta de crédito y el $74 \%$ mencionó no contar con ella.

\section{Instrumento}

Se definió al locus de control financiero como el grado en que un individuo considera que sus finanzas personales son contingentes con sus acciones, es decir, la conducta de obtener dinero puede ser controlada de manera interna, puede ser debida a causas afectivas o bien puede ser determinada por factores externos.

Para evaluar este constructo se redactaron 9 aseveraciones con cinco opciones de respuesta cerrada tipo Likert que van de completamente de acuerdo (1) a completamente en desacuerdo (5). Se escribieron las afirmaciones del locus de control financiero considerando los tres principales factores encontrados en México: Locus financiero de control interno (p.e. Yo decido cuando tener dinero), locus financiero de control externo (p.e. Tener dinero depende del destino) y locus financiero afectivo (p.e. Conseguir dinero depende de cómo le caiga a la gente). Los ítems se redactaron considerando que su contenido fuera congruente con la aproximación de aprendizaje social (Bandura, 2005; Rotter, 1975; Rotter, Chance \& Phares, 1972) y que pudieran reflejar las contingencias por las cuales las personas consideran que pueden tener dinero, para así obtener su validez de contenido.

Adicionalmente el instrumento incluía datos sociodemográficos referentes al ser hombre o mujer, la edad, el tipo de universidad a la que pertenecen (pública o privada), la carrera que estudian, su actividad principal (estudiar, trabajar o ambas), la percepción de su eficiencia (laboral y/o académica) y una pregunta sobre el manejo del crédito (tener o no tarjera de crédito).

\section{Procedimiento}

Se aplicó el instrumento a los universitarios de manera grupal, utilizando a los grupos naturales de clase para tal fin. Se les pidió que respondieran a un cuestionario elaborado para conocer algunas actividades relacionadas con las finanzas que realizan los universitarios. se les solicitó que respondieran de forma sincera, explicándoles que sus respuestas se utilizarían para fines de investigación.

\section{Consiedraciones éticas}

A todos los participantes se les aclaró que su participación era voluntaria y que la información era anónima, se les garantizó la confidencialidad de los datos proporcionados y se resolvieron las dudas que tuvieran. Se utilizó el consentimiento informado de los participantes y de las autoridades. El protocolo de investigación fue establecido de acuerdo al Reglamento de la Ley General de Salud, en su apartado sobre investigación en seres humanos (Secretaría de Salud, 2011).

\section{Resultados}

Para evaluar las características psicométricas del instrumento de control financiero propuesto para este estudio, se revisó la correlación ítem-total de los reactivos dentro de su respectiva dimensión. Se encontraron relaciones de bajas a moderadas de cada ítem dentro de su respectiva dimensión (interno, afectivo y externo) del control financiero. Para obtener la validez de contenido, además de incluir la correlación item total, se consideró la claridad conceptual de cada dimensión.

Para estudiar la validez de constructo del instrumento empleado, se realizó un análisis factorial exploratorio de ejes principales con rotación ortogonal y forzado a tres factores, para obtener la agrupación de los ítems como se ha propuesto en estudios previos (Correa et al., 2006; DíazLoving et al., 1984; García et al., 2000; La Rosa et al., 1986; Vera \& Cervantes, 2000). Se evaluó la adecuación de las matrices de correlaciones utilizando el índice de adecuación de la muestra de Kaiser-Meyer-Olkin (KMO) obteniendo un valor de 0.718 considerado como aceptable. El Índice de Esfericidad de Bartlett's fue significativo $\left(X^{2}=702.882 ; g l=36 ; p<.001\right)$ indicando la pertinencia de llevar a cabo el análisis factorial. 
Se consideró que el valor propio fuera superior a 1.0 (ver tabla 1). La solución factorial obtenida explica el $49.10 \%$ de la varianza total. El análisis de consistencia interna (coeficiente Alpha de Cronbach) para el total del instrumento mostró un índice de 0.62 (IC 95\% = .55 - .68).

Posteriormente, se realizaron correlaciones Rho de Spearman entre las interescalas del instrumento, para conocer el grado de asociación entre cada una de ellas. En la tabla 2, se observa que el control financiero interno se relaciona negativa y significativamente con el control financiero externo, no así con el control financiero afectivo que no muestra relación. El control financiero afectivo se relaciona positiva y significativamente con el control financiero externo.

La tabla 3 muestra la estadística descriptiva de las subescalas, en donde se observa que la mayor puntuación es para el control financiero interno, seguido del control financiero afectivo y por último, la puntuación promedio más baja la tiene el control financiero externo. En la misma tabla se aprecian los niveles de confiabilidad, en donde se destaca que el control financiero afectivo y el control financiero externo tienen una confiabilidad aceptable, no así para el control financiero interno que su nivel de fiabilidad se encuentra por debajo de lo permitido.

Se examinó la distribución normal del puntaje de cada factor del locus de control financiero, encontrandose que esta no fue estadísticamente normal de acuerdo a la prueba de KolmogorovSmirnov (K-S). El locus de control interno mostró una asímetría $=-.813$, una curtosis $=.622$ $\mathrm{y}$ un valor de K-S $(g l=300)=.138, p=.000$. El locus de control afectivo tuvo una asímetría $=.663$, una curtosis $=.049$ y un valor de K-S $(g l=300)=.111, p=.000$. El locus de control externo presentó una asímetría $=1.09$, una curtosis $=1.50 \mathrm{y}$ un valor de $\mathrm{K}-\mathrm{S}(g l=300)=.139$, $p=.000$. La existencia de la ausencia de normalidad en las distribuciones, es capital para asegurar la máxima discriminación interna de los sujetos con respecto al continuo subyacente del control financiero y para asegurar su continuidad métrica con los datos empíricos obtenidos.

Tabla 1.

Análisis factorial de la escala de locus de control financiero

\begin{tabular}{lccc}
\hline Ítems & $\begin{array}{r}\text { Control } \\
\text { afectivo }\end{array}$ & $\begin{array}{c}\text { Control } \\
\text { externo }\end{array}$ & $\begin{array}{c}\text { Control } \\
\text { interno }\end{array}$ \\
\cline { 2 - 4 } Conseguir dinero depende de lo agradable que soy & .888 & \\
Ser agradable con los demás me permitirá conseguir dinero & .655 & \\
Tener dinero depende de cómo le caiga a la gente & .572 & \\
Conseguir dinero depende del azar o la suerte & & .947 \\
Tener dinero depende del destino & & .657 \\
Tener dinero es responsabilidad del gobierno & & .344 & \\
Tener dinero depende de mí & & & .852 \\
Yo decido cuando tener dinero & & & .343 \\
Tener dinero depende del esfuerzo de las personas & & .283 \\
\hline Varianza explicada & $20.20 \%$ & $18.21 \%$ & $10.68 \%$ \\
\hline
\end{tabular}

Tabla 2.

Correlaciones interescala del locus de control financiero

\begin{tabular}{lccc}
\hline & Interno & Afectivo & Externo \\
\hline Locus de Control Financiero Interno & -- & .079 & $-.118^{*}$ \\
Locus de Control Financiero Afectivo & -- & $.464^{* *}$ \\
Locus de Control Financiero Externo & & & -- \\
\hline$* p<.05 * * p<.01 * * * p<.001$ & & &
\end{tabular}

Tabla 3.

Estadística descriptiva y confiabilidad de las subescalas

\begin{tabular}{lcccc}
\hline \multicolumn{1}{c}{ Escala } & Media & $\begin{array}{c}\text { Desviación } \\
\text { Estándar }\end{array}$ & $\alpha$ & $\begin{array}{c}\text { Número de } \\
\text { Ítems }\end{array}$ \\
\hline Locus de Control Financiero Interno & 3.99 & 0.7 & .471 & 3 \\
Locus de Control Financiero Afectivo & 2.18 & 0.9 & .767 & 3 \\
Locus de Control Financiero Externo & 1.88 & 0.8 & .732 & 3 \\
\hline
\end{tabular}


La tabla 4 muestra las comparaciones a través de la prueba $U$ de Mann-Whitney del locus de control financiero y las variables demográficas (sexo, tipo de carrera y universidad). Se encontraron diferencias estadisticamente significativas entre hombres y mujeres, los primeros puntúan más alto únicamente en el locus financiero afectivo comparado con las mujeres; es decir, que los hombres para obtener dinero utilizan las relaciones con las otras personas para conseguirlo. Los estudiantes de la universidad privada y los que son de las áreas económico-administrativas tienen un mayor locus financiero interno, es decir, este tipo de universitarios, decide el momento de obtener dinero y consideran que tener dinero depende de las acciones que ellos realicen para conseguirlo, comparado con los universitarios de la universidad pública o con un universitario que estudia en las áreas biológicas y sociales. Para el caso del locus financiero afectivo y externo, los universitarios obtuvieron puntuaciones de manera similar.

Adicionalmente, al llevar a cabo un análisis de varianza no paramétrico (Kruskal - Wallis), en- tre el tipo de control financiero y la ocupación, se encontraron diferencias estadísticamente significativas, en donde los universitarios que estudian y trabajan, tienen un mayor locus financiero interno comparado con los que sólo estudian y los que sólo trabajan tienen un mayor locus financiero afectivo comparado con los que estudian y trabajan (ver tabla 5).

$\mathrm{Al}$ obtener las diferencias en las variables financieras (uso de tarjeta de crédito) y laborales (clasificación de eficiencia en las cosas que realizan) se encontró que no existen diferencias estadísticamente significativas entre los universitarios que usan tarjeta de crédito y los que no usan, respecto a su control financiero, ya sea interno, afectivo o externo. Para conocer las diferencias en el nivel de eficiencia en actividades en el trabajo y/o académicas se formaron dos grupos (alta y baja eficiencia laboral y/o académica) a partir de la mediana. Se observa que quienes manifiestan tener mayor eficiencia en las actividades que realizan poseen un mayor control financiero interno, a diferencia de los de baja eficiencia. De manera contraria el

Tabla 4.

Diferencias en el locus de control financiero

\begin{tabular}{|c|c|c|c|c|c|}
\hline & \multicolumn{2}{|c|}{ Hombres } & \multicolumn{2}{|c|}{ Mujeres } & $\begin{array}{c}\text { Ude Mann- } \\
\text { Whitney }\end{array}$ \\
\hline & $M$ & $D E$ & $M$ & $D E$ & \\
\hline Locus financiero interno & 4.08 & 0.71 & 3.94 & 0.73 & 9.21 \\
\hline Locus financiero afectivo & 2.36 & 0.91 & 2.00 & 0.91 & $7.95 * * *$ \\
\hline \multirow[t]{3}{*}{ Locus financiero externo } & 1.96 & 0.89 & 1.84 & 0.77 & 9.81 \\
\hline & \multicolumn{2}{|c|}{ Universidad Pública } & \multicolumn{2}{|c|}{ Universidad Privada } & \\
\hline & $M$ & $D E$ & $M$ & $D E$ & $13.74 * *$ \\
\hline Locus financiero interno & 3.86 & 0.73 & 4.12 & 0.70 & $13.74 * *$ \\
\hline Locus financiero afectivo & 2.13 & 0.93 & 2.13 & 0.92 & 11.27 \\
\hline \multirow[t]{3}{*}{ Locus financiero externo } & 1.90 & 0.86 & 1.86 & 0.77 & 11.11 \\
\hline & \multicolumn{2}{|c|}{$\begin{array}{l}\text { Biológicas y } \\
\text { sociales }\end{array}$} & \multicolumn{2}{|c|}{$\begin{array}{l}\text { Económico } \\
\text { administrativas }\end{array}$} & \\
\hline & $M$ & $D E$ & $M$ & $D E$ & \\
\hline Locus financiero interno & 3.87 & 0.74 & 4.13 & 0.69 & $13.59 * *$ \\
\hline Locus financiero afectivo & 2.10 & 0.92 & 2.17 & 0.93 & 11.63 \\
\hline Locus financiero externo & 1.87 & 0.83 & 1.90 & 0.80 & 11.45 \\
\hline
\end{tabular}

$* * p<.01 * * * p<.001$

Tabla 5.

Diferencias en el control financiero por ocupación

\begin{tabular}{|c|c|c|c|c|c|c|c|}
\hline & \multicolumn{2}{|c|}{ Estudio } & \multicolumn{2}{|c|}{ Trabajo } & \multicolumn{2}{|c|}{$\begin{array}{c}\text { Estudio y } \\
\text { trabajo }\end{array}$} & \multirow[t]{2}{*}{$\begin{array}{c}\text { Kruskal - Wallis } \\
\left(X^{2}\right)\end{array}$} \\
\hline & $M$ & $D E$ & $M$ & $D E$ & $M$ & $D E$ & \\
\hline Locus financiero interno & 3.90 & 0.73 & 4.12 & 0.35 & 4.22 & 0.69 & $13.31 * *$ \\
\hline Locus financiero afectivo & 2.15 & 0.91 & 2.95 & 1.36 & 2.00 & 0.89 & 5.12 \\
\hline Locus financiero externo & 1.91 & 0.81 & 2.41 & 1.49 & 1.76 & 0.72 & 2.61 \\
\hline
\end{tabular}

${ }^{*} p<.05 * * p<.01 g l=2$ 
control financiero externo mostró diferencias significativas a favor de quienes consideran tener bajos niveles de eficiencia en las actividades que realizan, comparado con los de alto nivel de eficiencia (ver tabla 6), en otras palabras, quién es más eficiente posee un locus financiero interno y quién es menos eficiente cuenta con un locus financiero externo.

Para obtener la validez referida a un criterío se realizó una regresión logística para estimar la proporción del locus de control financiero en el nivel de eficiencia (alto o bajo) en las actividades (laboral y/o académico) que realizan los jóvenes. Los resultados obtenidos mostraron que el control financiero interno se estimó 1.6 veces en mayor proporción para los jóvenes de alta eficiencia respecto a los de baja eficiencia $(\mathrm{OR}=1.6, \mathrm{IC} 95 \%$ $=0.9-2.8, p<.05$ ); con un porcentaje correcto de clasificación del $92.3 \%$ y una pseudo R2 de Negelkerke $=0.10$. Considerando el porcentaje de varianza obtenida, el modelo sugiere como una posible explicación que quienes se consideran altamente eficientes en las actividades que realizan tienen mayor probabilidad de generar dinero por sí mismos.

\section{Discusión}

La evaluación del control que tengan las personas sobre el dinero fue medido por medio de factores que describen un conjunto de acciones para obtenerlo. La medición del constructo de locus de control en este estudio mostró que es posible medirlo a través de tres factores que se presentan de forma consistente en México. Derivado del análisis factorial exploratorio se pue- de observar que el primer factor obtenido, adjudica el logro del dinero a causas afectivas (locus de control financiero afectivo); el segundo factor alude a causas externas como la suerte (locus de control financiero externo); el último factor señala que tener dinero es atribuido a la decisión propia (locus de control financiero interno). La estructura de estos tres factores es similar a lo encontrado en otros estudios (Correa et al., 2006; Díaz- Loving et al., 1984; García et al., 2000; La Rosa et al.,1986; Vera et al., 2000) quienes encuentran dimensiones referentes a las causas por las que las personas adjudican la responsabilidad de su conducta.

Es relevante señalar que respecto al análisis factorial, este mostró la estructura reportada previamente en México (Correa et al., 2006; DíazLoving et al., 1984; García et al., 2000; La Rosa et al., 1986; Vera et al., 2000), sin embargo, el tercer ítem del locus de control financiero interno tiene un peso estructural bajo, a pesar de lo anterior, se decidió conservarlo considerando su pertinencia teórica. Este hallazgo indica la pertinencia de revisar el significado del ítem, así como la inclusión de nuevos ítems que reflejen con mayor precisión el constructo.

Considerando que el factor de control financiero interno tiene dificultades en su peso factorial, parece conveniente reflexionar sobre algunos inconvenientes que se presentaron en el instrumento y que se tendrán que solventar para futuros estudios.

1) Incluir un mayor número de reactivos para explorar con mayor profundidad las diferentes dimensiones del locus de control como se ha encontrado en estudios previos en México (García et al., 2000; La Rosa et al., 1986; Vera \& Cervantes, 2000).

Tabla 6.

Diferencias en el locus de control financiero y variables financieras

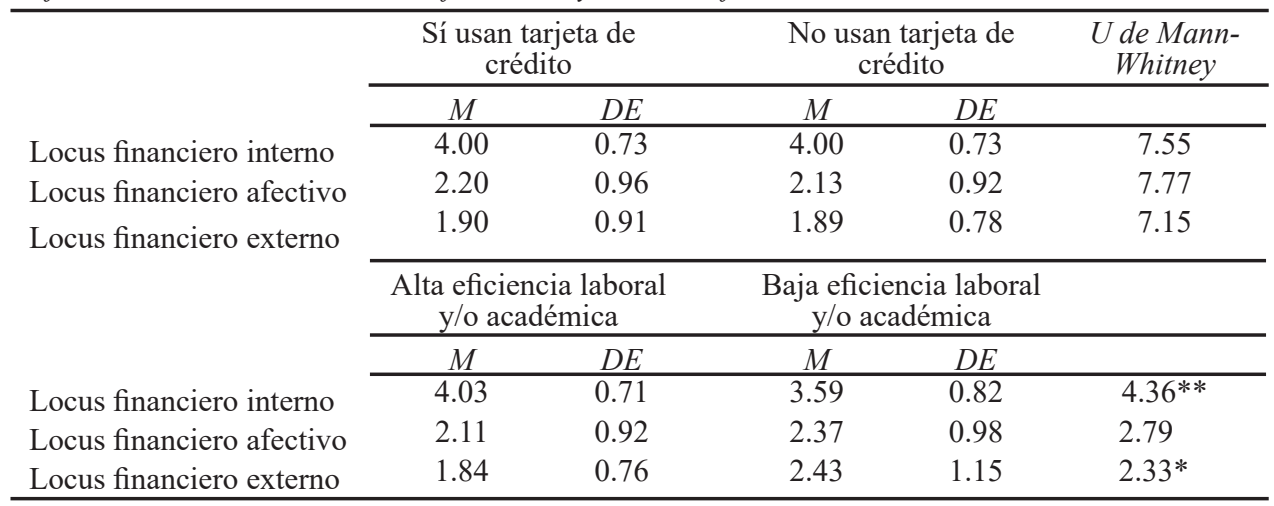

$* p<.05 * * p<.01 * * * p<.001$ 
2) Considerar reagrupar los reactivos de diferente manera, por ejemplo considerar únicamente dos dimensiones del locus de control (interno y externo). Aunque no se reporta en el estudio, se puso a prueba un nuevo análisis factorial bajo esta última idea, el resultado mostró claridad en la estructura del locus de control financiero afectivo y para el locus de control financiero interno, no así para el locus de control financiero externo, que se mezclo con el locus de control afectivo, lo que parece indicar que en una estructura factorial de sólo dos factores, se estaría obteniendo un locus de control externo orientado al locus financiero afiliativo, más que uno orientado a la externalidad exclusiva, como se mostró en las correlaciones realizadas (ver tabla 2). Con la obtención de ésta evidencia, desde nuestra perspectiva, la alternativa a seguir en un futuro es incrementar dimensionalidad al constructo de locus de control financiero encaminado a incorporar una cuarta dimensión del locus de control financiero pero de tipo social, de manera que la falta de consistencia del ítem "tener dinero depende del esfuerzo de las personas" podría entenderse que la palabra "personas" se refiere a "otras personas", lo que llevaría a que "tener dinero depende del esfuerzo de las (otras) personas" como amigos o familiares, por lo que en un futuro podría redactarse como un ítem de locus externo y no de locus interno o aún mejor como un ítem de locus financiero de control social. Estas consideraciones deberán de atenderse para trabajos futuros.

En cuanto al análisis de consistencia interna, la escala obtuvo un Alpha de Cronbach de .62 para el total del instrumento, que es considerado un nivel bajo de confiabilidad, por lo que se deberán revisar los reactivos de cada factor con la finalidad de mejorar su contenido, aumentar su fiabilidad e incorporar una mayor variedad de comportamientos vinculados con las contingencias internas, externas o afectivas (un mínimo de cinco ítems por factor) para conseguir dinero entre las personas. Se requiere aumentar los niveles de confiabilidad de cada factor, específicamente en el factor del control interno, sobre todo si se pretende vincular la escala obtenida con variables de la psicología económica o bien con variables psicosociales.

La escala presentada en este estudio puede ser una herramienta inicial para caracterizar la forma en la que los universitarios en México consideran el modo más viable de obtener dinero. Esta medición permite tener una comprensión más detallada de la obtención del dinero en este segmento poblacional. Desde esta perspectiva la investigación realizada en este estudio extiende la validez de constructo del locus de control interno, externo y afectivo, pero ahora aplicada a la forma de obtener dinero.

Los niveles de correlación interescalas, mostraron que el control financiero de tipo afectivo y el control financiero externo, se encuentran vinculados, es decir, para los universitarios que se encuentran en situaciones en las que conseguir dinero depende de cómo le caigan a la gente (control afectivo), es cuestión del destino o la suerte para conseguirlo (control externo), o el hecho de agradar a las personas es una forma externa para obtener dinero. La correlación negativa del control interno, con el control externo parece indicar que cuando las personas utilizan su propio esfuerzo para tener dinero (control interno), consideran que obtener dinero por medio de la suerte o el destino no es contingente con sus acciones, por lo que disminuye esperar obtenerlo por este medio. Lo obtenido respecto a la relación entre el locus de control externo y el locus afiliativo, parece encontrar apoyo en las características de la cultura mexicana (Díaz-Loving \& Andrade, 1984), en las que se describe la manipulación del ambiente mediante las capacidades afiliativas y comunicativas del sujeto. Este control afiliativo exige un tipo de enfrentamiento compatible con el estilo automodificador del mexicano (DíazGuerrero, 1994). Además, Kimble et al. (2002), mencionan que manipular el ambiente mediante otros, equivale a controlar nuestro destino, es decir a un control afectivo.

Adicionalmente, al realizar el análisis de medias de cada subescala, los universitarios obtuvieron la mayor puntuación en el locus de control financiero interno (3.99), seguido del control afectivo (2.18) y por último el locus externo (1.88). Los resultados obtenidos tanto para las correlaciones interescala como para el análisis de medias, parecen indicar que a pesar de tener la media más alta en el control financiero interno, las contingencias de aprendizaje para obtener dinero de los universitarios mexicanos (al menos en esta muestra), presenta una tendencia externa y socio-afectiva. Dichos resultados (mayor puntuación del control financiero interno) apoya lo señalado por Perry y Morris, 2005, respecto a te- 
ner mejor conducta financiera en quienes tienen locus de control interno, y parece contradecir las conclusiones de algunos autores (Díaz- Guerrero, 1994; García et al., 2000; Laborín et al., 2008; Triandis et al., 2002) al señalar que los países con culturas colectivistas tienen un locus de control externo. Lo anterior parece indicar que, la sociedad juvenil mexicana representada en esta muestra por estudiantes universitarios, cuando se encuentran bajo determinadas situaciones para conseguir dinero, perciben que las contigencias internas controlan de mejor manera su forma de obtener dinero.

El perfil comparativo del locus de control financiero en los universitarios indica que el control financiero afectivo es más alto en los hombres universitarios, es decir, los hombres consideran que para tener dinero es necesario utilizar sus habilidades afiliativas y comunicativas con las demás personas con la finalidad de ganar dinero. Lo anterior concuerda con los resultados arrojados por Haider et al. (2013), así como con lo señalado por Denegri et al. (2012) quienes mencionan que el género es uno de los predictores significativos que permiten discriminar entre aquellos que usan tarjetas de crédito y que se encuentran más endeudados, en comparación con aquellos que tienen menos tarjetas de crédito y están menos endeudados.

Las diferencias del control financiero interno a favor de la universidad privada y de las áreas económico- administrativas, se traduce en que los universitarios de estas áreas de conocimiento han recibido una formación bajo la cual perciben que son capaces de controlar las contingencias que elicitan obtener dinero por cuenta propia, además de tener la capacidad de decidir el momento adecuado para ganar dinero, así como de atribuir su éxito financiero a sus esfuerzos y capacidades, todo ello es contrario a lo que realizan los universitarios de escuelas públicas al tener una formación vinculada con áreas sociales y de la salud; lo anterior corrobora su importancia y su estudio posterior con el uso de instrumentos de crédito que se le otorguen a los universitarios, ya sea de áreas administrativas o económicas o bien de áreas sociales y de la salud, en estos últimos se deberá centrar la atención ya que las políticas universitarias lo podrían usar a favor de generar mejores niveles económicos o bien como lo señalan Denigri et al. (2012) aumentar progresivamente sus patrones diferenciales de endeuda- miento con la edad, en quienes puede coexistir fuentes formales de endeudamiento (comercios y tarjetas de crédito) e informales como son los amigos y la familia.

En la transición de ser estudiante o ser estudiante y trabajar al mismo tiempo, a ser únicamente trabajador ya graduado, existe una transformación en los valores, expectativas personales o contingencias en la forma de conseguir dinero, es decir, los universitarios que estudian y trabajan, tienen un control financiero interno al atribuir que tener dinero depende del esfuerzo de ellos mismos y que se ve reforzado al realizar dos actividades (estudiar y trabajar) al mismo tiempo. En el caso de los universitarios que ya sólo trabajan han desplazado la utilidad exclusiva de un control interno a un control financiero afectivo, es decir, atribuyen su éxito financiero a la relaciones sociales que establecen con quién se relaciona el individuo, en contextos o situaciónes particulares (Díaz-Guerrero, 1994; Laborín et al., 2008; Palacios, 2011; Vera \& Cervantes, 2000) como puede ser en este caso el laboral. Lo anterior se encuentra parcialmente apoyado en lo que señalan estudios realizados en poblaciones universitarias (Amar et al., 2007; Boddinton \& Kemp, 1999; Denegri et al., 2012; Palacios, 2014; Rodríguez, 2006) quienes muestran la influencia potencial que tienen las expectativas de futuro laboral. Esto implica que para los estudiantes universitarios el endeudamiento actual puede ser considerado como una situación transitoria que les permite sustentar su actual estilo de vida, pero que sería superado una vez que aumenten sus ingresos al egresar de la universidad.

Al obtener las diferencias en las variables financieras (uso de tarjeta de crédito y clasificación de eficiencia en las cosas que realizan), se encontró que no existen diferencias entre los universitarios que usan tarjeta de crédito respecto a su control financiero, ya sea interno, afectivo o externo. Mientras que para el nivel de eficiencia en las actividades que realizan poseen un mayor control financiero interno, es decir, consideran que obtener dinero depende de ellos mismos. Lo anterior abre dos posibilidades, la primera señala que en esta muestra la obtención de dinero depende exclusivamente de su eficiencia para lograrlo y la segunda, que usar una tarjeta de crédito no depende en exclusiva de acciones contingentes para lograr más dinero. La última posibilidad requiere de mayor investigación en esta área para 
tener claridad del uso y comprensión que le dan los universitarios a las tarjetas de crédito.

Los resultados encontrados tienen implicaciones para la personalidad de los mexicanos, debido a que la aplicación y desarrollo del locus de control, está influido por factores socioculturales (Correa et al., 2006; Díaz-Guerrero, 1994; Díaz- Loving et al., 1984; García et al., 2000; La Rosa et al., 1986; Palacios et al., 2011; Vera et al., 2000), que elicítan la forma en que se percibe que los reforzadores son contingentes con la conducta. En este sentido, en un futuro se podrá indagar el efecto que tienen los factores socioculturales en el locus de control financiero. La investigación previa realizada en México (Correa et al., 2006; Díaz- Guerrero, 1994; Díaz- Loving \& Andrade, 1984; La Rosa et al., 1986; Rotter, 1975, 1990; Vera et al., 2000) ha mostrado diferencias en las contingencias de reforzamiento, ya sean producidas por la propia conducta (control interno) o bien por contingencias de reforzamiento determinadas por la suerte o los otros (control externo) así como por contingencias de reforzamiento afectivas (control afectivo).

Es importante señalar una serie de limitaciones del estudio, una de ellas posiblemente la más importante se encuentra en el instrumento, particularmente por los bajos niveles de confiabilidad en el factor interno, por lo que en próximos estudios será necesario incrementar el número de ítems a fin de mejorar la consistencia interna del mismo, con la finalidad de obtener resultados más certeros y fiables. Otra limitación tiene que ver con el tamaño de la muestra, por lo que para futuros estudios se tendrá que ampliar la misma, así como replicar el estudio en diversas muestras con la finalidad de corroborar la estructura teórica del instrumento de locus de control financiero, su fiabilidad y su vinculación con la conducta económica. Así mismo, al tratarse de un estudio preliminar, las conclusiones tendrían que tomarse con mayor cautela considerando que los resultados todavía no son definitivos, por lo que su aplicación tendrá un menor alcance.

Debido a las dificultades psicométricas encontradas en el instrumento, se abren nuevas preguntas de investigación, por ejemplo, ¿es conveniente construir una nueva escala que mida locus de control financiero? o ¿podría adaptarse otra ya existente que cuente con buenas propiedades psicométricas? Tres respuestas se pueden encontrar a estas interrogantes. La primera, es seguir utilizando la escala presentada en este estudio sin modificaciones, lo que consideramos no es lo más conveniente. Segundo, revisar los resultados obtenidos en el presente estudio y ajustar lo necesario a fin de mejorar el instrumento y tercero, optar por realizar la adaptación de otra escala ya existente que cuente con adecuadas propiedades psicométricas para medir el locus de control financiero en la cultura mexicana.

A modo de conclusión se puede señalar que lo encontrado en el presente estudio indica que los jóvenes difieren en el valor del reforzador, dependiendo de las situaciones bajo las cuales ellos perciben como contingente obtener dinero o no hacerlo. Pero estas situaciones se ven diferenciadas por aspectos socio-demográficos, como son ser hombre, ser estudiante de una universidad privada, estudiar carreras de tipo económico- adminsitrativas y ser eficientes en las actividades que realizan, son aspectos distinguibles del locus de control con el que cuenten, es decir, un locus de control interno y afectivo, con lo cual, los jóvenes realizan la conducta de obtener dinero en función de su propia decisión para obtenerlo.

\section{Referencias}

Amar, J., Abello, R., Denegri, M., \& Llanos, M. (2007).Pensamiento económico en jóvenes universitarios. Revista Latinoamericana de Psicología, 39(2), 363-373.

Alejo, R. A., Rojas, A. P., \& Pérez-Acosta, A. (2008). Psicología y asuntos económicos: una aproximación al estado del arte. International Journal of Psychological Research, 1(1), 49-57.

Ardila, R. (2011). El mundo de la psicología. Bogotá: Manual Moderno.

Bandura, A. (1994). Social cognitive theory and exercise of control over HIV infection. In: DiClemente, R. J. and Peterson, J. L. (Eds.). Preventing AIDS theories and methods of behavioral interventions (pp. 89-116). New York: Plenum Press.

Bandura, A. (2001). Social Cognitive Theory: An agentic perspective. Annual Review of Psychology, 52, 1-26.

Bandura, A. (2002). Environmental sustainability by sociocognitive deceleration of population growth. En P. Schmuck \& W. Schultz (Eds.). Psychology of Sustainable Development (pp. 209-238) Massachussetts: Kluwer Academic Publishers.

Bandura, A. (2005). Guide for constructing self-efficacy scales. En: Pajares, F. \& Urdan, T. (Ed.). Self-efficacy, belief of adolescents (pp. 307-337). USA: Age Publishing.

Bedolla, R. B., \& Mena, G. J. (2004). Relación de voto duro con autoeficacia y locus de control. La Psicología Social en México, 10, 563-569.

Baguma, P., \& Chireshe, R. (2012). Predictors of economic locus of control among university students in Uganda. Journal of Psychology in Africa, 22(2), 279-282. 
Boddinton, L., \& Kemp, S. (1999). Student debt, attitudes towards debt, impulsive buying, and financial management. NewZealandJournal of Psychology, 28(2), 89-93.

Bustos, A, J. M., Flores, H. M., \& Andrade, P. P. (2004). Predicción de la conservación de agua a partir de factores socio-cognitivos. Medio Ambiente y Comportamiento Humano, 5 (1 y 2), 53-70.

Camacho, V. M., Moreno, C. S., \& Hernández, R. A. (1994). Locus de control, escolaridad de los padres y rendimiento académico en adolescentes. La Psicología Social en México, 5, 31- 36.

Cázares, C. A., \& Berridi, R. R. (2000). Multidimensionalidad del locus de control a los 8 y 9 años de edad. $L a$ Psicología Social en México, 8, 121- 126.

Correa, R. F., Bedolla, R. B., \& Reyes, L. I. (2006). Estructura del control personal. En: Sánchez, A. R., DíazLoving, R. y Rivera, A. S. (Eds.). La Psicología Social en México (pp. 66- 72), Vol. 11, México: AMEPSO.

Crusius, J., van Horen, F., \& Mussweiler, T. (2012). Why process matters: A social cognition perspective on economic behavior. Journal of Economic Psychology, 33, 677- 885

Cruz, J. (2001). Psicología económica. Suma Psicológica, $8(2), 213-236$.

Denegri, C. M., Cabezas, G. D., Del Valle R., C., González, G., Y., \& Sepúlveda, A. J. (2012). Escala de actitudes hacia el endeudamiento: validez factorial y perfiles actitudinales en estudiantes universitarios chilenos. Universitas Psychologica, 11(2), 489-509.

Díaz -Guerrero, R. (1994). La Psicología del mexicano (6a ed.). México: Trillas.

Díaz- Loving, R., \& Andrade, P. P. (1984). Una escala de locus de control para niños mexicanos. Revista Interamericana de Psicología, $18(1$ y 2), 21-33.

Dijkstra, A., \& De Vries, H. (2000). Clusters of precontemplating smokers defined by the perceptions of the pros, cons and self-efficacy. Addictive Behaviors, 25(3), 373- 385 .

Furnham, A. (1986). Economic locus of control. Human Relations, 39(1), 29-43.

Galindo, O., \& Ardila, R. (2012). Psicología y pobreza. Papel del locus de control, la autoeficacia y la indefensión aprendida. Avances en Psicología Latinoamericana, 30(2), 381-407.

García, C. T., \& Reyes, L. I. (2000). Estructura del locus de control en México. La Psicología Social en México, 8, 158-164.

González, L. D., Corral, V. V., \& Maytorena, M. A. (2002). Modelo estructural de locus de control escolar. La Psicología Social en México, 9, 276-281.

Gwaltney, C., Shiffman, S., Norman, G., Paty, Kassel, J., Gnys, M., Hickcox, M., \& Balanbis, M. (2001). Does smoking abstinence self efficacy vary across situations? Identifying context- specificity with the relapse situation efficacy questionnaire. Journal of Consulting and Clinical Psychology, 66, 516- 527.

Haider, Z., I., \& Naeem M. (2013). Locus of control in graduation students. International Journal of Psychological Research, 6(1), 15-20.

Kimble, C., Hirt, E., Díaz-Loving, R., Hosh, H., Lucker, G. W., \& Zárate, M. (2002). Psicología Social de las Américas. México: Pearson Educación.

La Rosa, J., Díaz- Loving, R., \& Andrade, P. P. (1986). Escalas de locus de control: problemas y contribuciones. Revista Mexicana de Psicología, 3(2), 150-153.
Laborín, Á. J., Vera. N. J., Durazo, S. F., \& Parra, A. E. (2008). Composición del locus de control en dos ciudades latinoamericanas. Psicología desde el Caribe, 22, 63-83.

Lachman, M., \& Weaver, S. (1998). The sense of control as moderator of social class differences in health and well-being. Journal of Personality and Social Psychology, 74, 763-773.

Leong, J., Molassiotis, A., \& Marsh, H. (2004). Adherence to health recommendations after a cardiac rehabilitation program in post-myocardial infarction patients: the role of health beliefs, locus of control and psychological status. Clinical Effectiveness in Nursing, 8(1), 26-38. doi: 10.1016/j.cein.2004.02.001

Lindstrom, M., \& Rosvall, M. (2014). Economic stress and lack of internal health locus de control: A life course approach. Scandinavian Journal of Public Health, 42(1), 74-81. doi: 10.1177/1403494813504503

Lipina, S. J., \& Colombo, J. A. (2009). Poverty and brain development during childhood: An approach from cognitive psychology and neuroscience. Washington, D. C: American Psychological Association.

Montero, S, N., Rivera, A. S., Reyes, L. I., \& Díaz -Lovíng, R. (2008). Adaptación de la escala de locus de control para parejas.. En: Rivera, A. S., Díaz-Loving, R. Sánchez, A. R. y Reyes, L. I (Eds.). La Psicología Social en México (pp. 621-624), Vol. 12, México: AMEPSO.

Palacios, D. J. (2010). Autoeficacia e intención conductual del consumo de tabaco en adolescentes: validez factorial y relación estructural. Adicciones, 22(4), 325-330.

Palacios, D. J. (2011). Las conductas de riesgo del adolescente. México: Centro de Investigación e Innovación Biopsicosocial, AC.

Palacios, D. J. (2014, Junio). Psicología económica y comportamiento de consumo. Segundo Coloquio de Psicología efectuado en la Universidad Humanitas, México.

Palacios, D. J. (2015). Estimación psicométrica de la escala de autoeficacia ante conductas de riesgo para adolescentes en México. Psychosocial Intervention, 1, 1-7.

Palacios,D.J.\&Bustos,A.J.M.(2012a). Modelodeautoeficacia y habilidades ambientales como predictores de la intencióny disposición proambientalenjóvenes. RevistaIntercontinental de Psicología y Educación, 14(2), 143- 163.

Palacios, D. J., \& Bustos, A. J. M. (2012b). La teoría como promotor para el desarrollo de intervenciones psicoambientales. Psychosocial Intervention, 21(3), 245- 257

Palacios, D. J., \& Bustos, A. J. M. (2013). Validez factorialdela autoeficacia ambiental y su influencia estructural sobre la conducta proambiental en jóvenes. Revista Iberoamericana de Evaluación en Psicología, 35(1), 95-111.

Palacios, J. R., Bustos, J. M., \& Soler, A. L. (2016). Personalidad en diferentes niveles del comportamiento de compra. En: Rivera, A. S., Díaz-Loving, R., Reyes, L. I. y Flores, G. M. (Eds.). Aportaciones Actuales de la Psicología Social (pp. 414- 420), Volumen III. México: AMEPSO. ISBN: 978-607-96539-1-0.

Palacios, D. J., \& Martínez, M. R. (2017). Descripción de características de personalidad y dimensiones socioculturales en jóvenes mexicanos. Revista de Psicología, 35(2), 453- 484.

Palacios, D. J., \& Parrao, L. M. (2010). Intención, habilidades y eficacia para predecir el uso del condón. En: Rivera, A. S., Díaz-Loving, R. Sánchez, A. R., y Reyes, L. I. (Eds.). La Psicología Social en México (pp. 267- 272), Vol. 13, México: AMEPSO. 
Palacios, D. J., \& Ramírez, A. V. (2016). Estudio comparativo de la autoeficacia saludable en las conductas alimenticias de riesgo. Revista Psicología Iberoamericana $24(2), 17-25$.

Perry, V. G., \& Morris, M. D. (2005). Who is in control? The role of self perception, knowledge, and income in explaining consumer financial Behavior. Journal of Consumer Affairs, 39(2), 299-313.

Plunkett, H. R., \& Buehner, M. J. (2007). The relation of general and specific locus of control to intertemporal monetary Choice. Personality and Individual Differences, 42, 1233-1242. doi:10.1016/j.paid.2006.10.002

Quintanilla, P. I. (1998). La psicología económica y del consumidor en España. Reflexiones conceptuales y práctica profesional. Papeles del Psicólogo, 70, 48- 54.

Rodríguez, V. J. (2006). Validación del modelo psicoeconómico del consumidor. Pensamiento y gestión, 20, 1-54.

Rotter, J. B. (1966). Generalized expectancies for internal versus external control of reinforcement. Psychological Monographs, 80, 1-26.

Rotter, J. B. (1975). Some problems and misconceptions related to the construct of internal versus external control of reinforcement. Journal of Consulting and Clinical Psychology, 43(1), 56-67. doi: 10.1037/h0076301

Rotter, J. B. (1990). Internal versus external control of reinforcement: A case history of a variable. American Psychologist, 45(4), 489-493.

Rotter, J. B. \& Mulry, R. C.(1965). Internal versus external control of reinforcement and decision time. Journal of Personality and Social Psychology, 2(4), 598-604. doi: 10.1037/h0022473

Rotter, J. B., Chance, J. E., \& Phares, E. J. (1972). Applications of a social learning theory of personality. England: Oxford, Holt, Rinehart \& Winston.
Riccio-Howe, L. (1991). Health values, locus of control, and cues to action as predictors of adolescent safety belt use. Journal of Adolescent Health, 12(3), 256-262.

Rowley, M., Lown, J., \& Piercy, K. (2012). Motivating Women to Adopt Positive Financial Behaviors. Journal of Financial Counseling and Planning, 23(1), 48-62.

Samuel-Lajeunesse, J. F., \& Gil Juárez, A. (2014). Psicología económica y del comportamiento del consumidor. España: Editorial UOC.

Secretaría de Salud. (2011). Reglamento de la Ley General de Salud en Materia de Investigación para la Salud. Recuperado de http://www.salud.gob.mx/unidades/ cdi/nom/compi/rlgsmis.html

Stenström, U., \& Andersson, P. (2000). Smoking, blood glucose control, and locus of control beliefs in people with Type 1 diabetes mellitus. Diabetes Research and Clinical Practice, 50, 103-107. doi: 10.1016/S01688227(00)00169-8

Stuart, K., Borland, R., \& McMurray, N. (1994). Self-efficacy, health locus of control, and smoking cessation. Addictive Behaviors, 19(1), 1-12.

Triandis, H., \& Suh, E. (2002). Cultural influences on personality. Annual Review of Psychology, 53,133-160. doi. org/10.1146/annurev.psych.53.100901.135200

Vera, J., \& Cervantes, N. (2000). Locus de control en una muestra de residentes del noroeste de México. Psicología y Salud, 10(2), 237-247.

Wilson-Barlow, L., Hollins, T., \& Clopton, J. (2014). Construction and validation of the healthy eating and weight self-efficacy (HEWSE) scale. Eating Behaviors 15, 490-492. doi: 10.1016/j.eatbeh.2014.06.004. 